\title{
Educación abierta e innovación en TISP: diseño de un curso MOOC sobre Iniciación a la Traducción e Interpretación en los Servicios Públicos \\ Open Education and Innovation in PSIT: Designing a MOOC on Introduction to Public Service Interpreting and Translation
}

\author{
Carmen Valero-Garcés ${ }^{1}$, Bianca Vitalaru², Raquel Lázaro Gutiérrez ${ }^{3}$ \\ carmen.valero@uah.es, bianca.vitalaru@gmail.com, raquel.lazaro@uah.es
}

\author{
${ }^{1}$ Filología Moderna \\ Universidad de Alcalá \\ Alcalá de Henares, España
}

\author{
${ }^{2}$ Filología Moderna \\ Universidad de Alcalá \\ Alcalá de Henares, España
}

\author{
${ }^{3}$ Filología Moderna \\ Universidad de Alcalá \\ Alcalá de Henares, España
}

\begin{abstract}
Resumen- En este artículo se describe el curso en línea masivo y abierto (MOOC) “¡Iníciate a la Traducción e Interpretación en los Servicios Públicos!” de la Universidad de Alcalá, el primer curso sobre iniciación a la Traducción e Interpretación en los Servicios Públicos (TISP) en España, así como los resultados de calidad obtenidos hasta este momento a través de encuestas de satisfacción considerando la variedad de los perfiles del alumnado. A lo largo de diez semanas y utilizando el español como lengua vehicular, el curso ofrece una aproximación al mundo de la TISP y pretende contribuir a la difusión de información sobre esta profesión emergente entre profesionales y usuarios de distintos sectores en los que la comunicación intercultural se encuentra presente.
\end{abstract}

\section{Palabras clave: Educación abierta, MOOC, TISP.}

Abstract- This article describes the Massive Open Online Course (MOOC)“Try Public Service Interpreting and Translation!” taught at the University of Alcalá, the first course on introduction to Public Service Interpreting and Translation (PSIT) in Spain, as well as the results obtained up to now from quality surveys considering the variety of students' profiles who attended it. The course, in Spanish, offers a general overview of the world of PSIT over a period of ten weeks. It aims to contribute to the dissemination of information about this emerging profession among professionals and users of different sectors who need specific knowledge on intercultural communication.

Keywords: Open Education, MOOC, PSIT.

\section{INTRODUCCIÓN}

Con la llegada de las nuevas tecnologías y sus constantes avances estamos asistiendo a un proceso de cambio e innovación en los conceptos tradicionales de enseñanza y aprendizaje. La introducción de los MOOC (Massive Online Open Course) como cursos gratuitos y abiertos al público en el contexto de la formación online o en red, rompe las barreras del aula como espacio físico cerrado y abre un nuevo horizonte de posibilidades formativas. No obstante, el desarrollo de materiales no evoluciona de forma uniforme para todas las ramas. A este respecto, Álvarez et al. (2016, p. 1) señalan que todavía no se han desarrollado experiencias MOOC significativas en el ámbito de la enseñanza de la traducción y, por extensión, podría añadirse la interpretación.

En lo que al territorio español se refiere, esta afirmación se refleja claramente en la ausencia de MOOC específicos en la Traducción e Interpretación a los Servicios Públicos (TISP), campo que empieza a adquirir una relevancia considerable debido al incremento de población extranjera y a su necesidad de comunicarse de forma efectiva con personal de instituciones públicas como juzgados, comisarías, hospitales, centros de salud, oficinas de inmigración o escuelas. Ante esta necesidad de materiales formativos en línea, los grupos FITISPos y FITISPos E-learning, de la Universidad de Alcalá, ambos dedicados, entre otros aspectos, a la formación y elaboración de materiales formativos, pretenden contribuir a eliminar esta laguna de conocimiento mediante la creación de MOOC. En este artículo nos centraremos en uno en concreto, dedicado al área de la TISP en España, donde, como ya mencionábamos, no existe actualmente un curso específico de estas características.

\section{CONTEXTO}

\subsection{ESTADO DE LA CUESTIÓN}

La utilización masiva que se hace de este tipo de herramienta a nivel global es una prueba más de la aplicación de las nuevas tecnologías para el diseño de contenidos formativos y para la enseñanza a través de cursos online gratuitos.

Según Roig, Mengual y Suárez (2014, p. 28) no existe un sistema ni un acuerdo generalizado con respecto a los diferentes aspectos importantes que intervienen en el diseño e incluso en la utilización de los MOOC en la enseñanza, llegando incluso a generarse polémicas al respecto. Concretamente, menciona aspectos como su clasificación, su 
papel en la enseñanza universitaria, su acreditación, su modalidad de enseñanza y cita una serie de estudios significativos para cada uno de ellos.

A su vez, destacamos las reflexiones y debates con respecto a la utilidad de los MOOC especialmente desde el punto de vista pedagógico en el proceso de formación y aprendizaje a nivel universitario, así como de su calidad, entre otros aspectos.

De los aspectos mejor valorados señalamos algunos como el desarrollo de determinadas competencias en los alumnos, la utilización del potencial de las nuevas tecnologías y su formato. Con respecto al primer tema, Roig, Mengual y Suárez (2014, pp. 30-37), en su estudio sobre la metodología de enseñanza utilizada y la calidad pedagógica de los MOOC, señalan competencias como la capacidad de trabajo en grupo, el pensamiento crítico y el potencial de la herramienta de uso de las redes sociales y nuevas tecnologías para la creación y publicación de recursos de aprendizaje. Por otro lado, otros autores, como Méndez García (2013, pp. 2), destacan el papel importante del alumno en su proceso formativo $\mathrm{y}$ en la creación de contenido, así como la relación entre profesor y alumnado: "desplazan la relación jerárquica entre profesor y alumno, de modo que el proceso de aprendizaje se reparte (de ahí las referencias en la literatura sobre MOOC a la idea de una 'responsabilidad distribuida' en el aprendizaje)". Finalmente, según Marco Cuenca, Arquero Avilés et al. (2010), el formato de estos cursos es bastante práctico para los posibles usuarios e incluso para las universidades, ya que facilita su promoción e imagen adaptada a las necesidades de la educación actual.

En cambio, algunos de los aspectos que se cuestionan por diferentes autores, como, por ejemplo, Cabero Almenara, Llorente Cejudo y Vázquez Martínez (2014, p. 21).

- $\quad$ Su utilidad pedagógica y falta de novedad ya que realmente se trata de educación a distancia.

- La falta de investigación con respecto a sus "posibilidades educativas y sus limitaciones y problemáticas” y la preocupación por los aspectos tecnológicos y el "modelo educativo" empleado.

- $\quad$ La verificación de la identidad de los alumnos.

- $\quad$ La gran tasa de abandono.

Como idea general destaca la falta de consenso y de estudios más profundos sobre diferentes aspectos relacionados con la utilidad formativa actual de los MOOC en general y de los MOOC relacionados de alguna manera con la traducción en particular.

\subsection{CONTEXTO DEL MOOC PROPUESTO}

“¡Iníciate a la Traducción e Interpretación en los Servicios Públicos!” (Nombre inicial “¡Atrévete a Traducir y a Interpretar en los Servicios Públicos!”) fue la primera propuesta de este curso MOOC y se presentó en la Comisión Europea - Dirección General de Traducción en octubre de 2016 dentro del congreso Translating Europe Forum (Álvaro Aranda y Valero-Garcés; 2017). Tras un proceso de revisión y pilotaje se lanzó a través de la plataforma virtual de la Universidad de Alcalá (información disponible en Youtube) y actualmente se está trabajando en su ampliación y adaptación a idiomas susceptibles de necesitar esta formación por el volumen de población migrante que generan (inglés y francés como lenguas puentes, además de chino, árabe y ruso).

El curso, de carácter introductorio, va dirigido a personas que sientan curiosidad por la comunicación intercultural, las figuras y funciones del traductor e intérprete que trabaja en el campo de los servicios públicos, los principales escenarios de actuación o los agentes involucrados en el proceso. Asimismo, pretende orientar a personal de ONG, bilingües o voluntarios que efectúan tareas de enlace con personas -inmigrantes, refugiados, víctimas de crisis o desastres naturales, etc.- que se dirigen a cualquier servicio público en busca de atención y que desconocen o no manejan satisfactoriamente la lengua de contacto — el español, en este caso.

Este curso MOOC responde a dos objetivos principales:

1. Contribuir a la formación de personas que actúan como enlace en las distintas instituciones de los servicios públicos.

2. Concienciar al público acerca de las particularidades de la TISP y la necesidad de formación específica en el ámbito. En este sentido, se persigue dotar al alumno de este curso con las bases necesarias para que determine si desea profundizar en la TISP a través de propuestas de formación más completas y específicas.

\section{DESCRIPCIÓN}

En este apartado describiremos la modalidad de acceso al curso, su estructura, así como la metodología de enseñanza y trabajo que debe seguir el alumno para el máximo aprovechamiento y reflexión sobre sus contenidos.

\subsection{DESCRIPCIÓN GENERAL: ACCESO Y ESTRUCTURA}

El curso, de acceso abierto, se encuentra alojado en la plataforma OpenEducation

(https://openeducation.blackboard.com) y no se exige ningún requisito inicial de acceso (Figura 1 ).

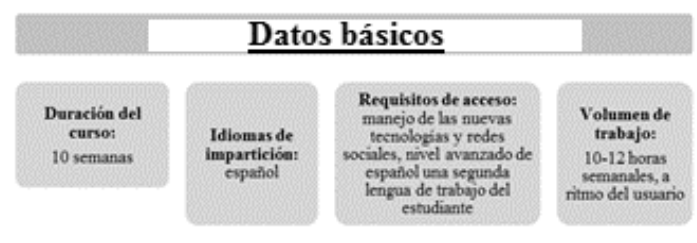

Figura 1: Datos básicos sobre el MOOC

Como se refleja en la Figura 2, el curso se encuentra estructurado en torno a tres bloques de conocimiento con una duración determinada, que se dividen en varios subapartados específicos y se presentan según su orden de dificultad y grado de especialización. 


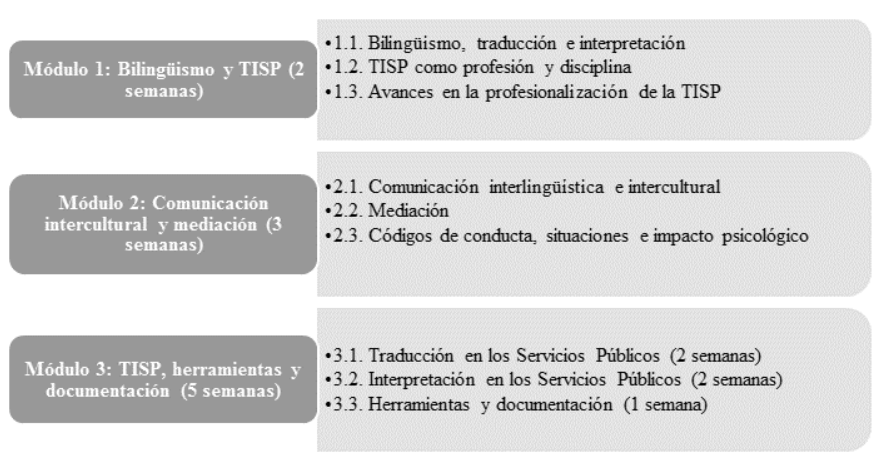

Figura 2: Distribución de contenidos

Cada uno de estos bloques incluye la exposición en formato vídeo de una serie de conceptos fundamentales asociados al tema en cuestión. Posteriormente, se invita a los alumnos a reflexionar mediante el intercambio de opiniones en el foro y la realización de actividades dirigidas al desarrollo de competencias y aptitudes específicas para la traducción y la interpretación en los servicios públicos.

\subsection{MÉTODOLOGÍA DE ENSEÑANZA Y TRABAJO DEL ALUMNO}

El curso persigue favorecer el aprendizaje autónomo y cooperativo de los estudiantes inscritos. Por lo tanto, las actividades constituyen una parte fundamental, ya que incitan al alumno a reflexionar sobre las nociones teóricas y proporcionan bibliografía para realizar búsquedas más profundas y alcanzar conclusiones propias. Los ejercicios se encuentran dirigidos al desarrollo de competencias y aptitudes específicas para la traducción y la interpretación en los servicios públicos: competencia lingüística e intercultural, competencia documental, competencia tecnológica y competencia temática (Red Másteres Europeos de Traducción EMT, 2009, p. 1-2).

Entre estas propuestas prácticas se incluyen actividades de formación y evaluación de diferentes tipos:

- Autoevaluaciones, con preguntas tipo verdadero/falso o de respuesta múltiple.

- Actividades en formato vídeo de búsqueda, lectura o reflexión, que incorporan las redes sociales (Twitter) y permiten el intercambio de percepciones y experiencias en el foro.

- Ejercicios de traducción o interpretación en los que se ilustran algunas de las principales dificultades (aspectos culturales, terminología, etc.) y que permiten poner en práctica habilidades específicas (toma de notas, memoria, capacidad de reacción, etc.).

A continuación, el texto que sigue (Figura 3) muestra una de las actividades disponibles:

\section{ACTIVIDAD 3.1.a}

Visita la página web de Salud Pública del Ayuntamiento de Madrid (www.madridsalud.es) y examina las publicaciones a las que pueden acceder los usuarios y los idiomas en que se encuentran disponibles. Selecciona uno de estos textos e imagina que debes traducirlo para un grupo de pacientes que pertenecen a otra comunidad y hablan otra lengua. ¿Qué elementos tendrías en cuenta? ¿Crees que tendrías que adaptar algunos aspectos| del texto porque pueden resultar problemáticos o tabú para el nuevo público? Comenta en esta sección las principales dificultades encontradas y los cambios que sugerirías.

Figura 3: Ejemplo de actividad de búsqueda, reflexión y debate

Con respecto al método de trabajo que debe seguir el alumno para poder tener acceso a la información y desarrollar las competencias, cabe señalar que el curso MOOC sigue una metodología estructurada e idéntica en los tres bloques de contenidos. Al pulsar sobre cada módulo, se despliegan una serie de carpetas que siguen un esquema fijo y se muestran de forma lógica. En primer lugar, se accede a un vídeo de presentación del módulo a nivel general en el que el usuario tiene la oportunidad de familiarizarse con un índice de los contenidos que van a tratarse durante la semana en cuestión, así como conocer los objetivos y habilidades que se desarrollarán en cada punto (Figura 4).

Presentación módulo 3: traducción e interpretación en los servicios públicos, herramientas y documentación

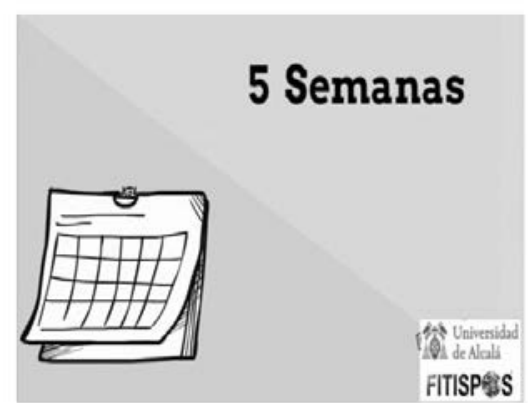

Figura 4: Ejemplo de vídeo de presentación del módulo 3

Posteriormente, el usuario navega a través de carpetas secundarias en las que se incluye la exposición en formato vídeo de los conceptos seleccionados para cada uno de los temas. Una vez visualizada la teoría, el alumno puede descargarse presentaciones PowerPoint que contienen las ideas clave, así como un documento Word en el que se incluyen las referencias bibliográficas mencionadas y enlaces de acceso abierto y directo a estas. Son éstos los pasos previos necesarios antes de iniciar la parte práctica, que pivota alrededor de actividades individuales y en grupo asociadas a cada tema $\mathrm{y}$, en último lugar, se requiere la realización de una breve autoevaluación a modo de repaso (Figura 5). 


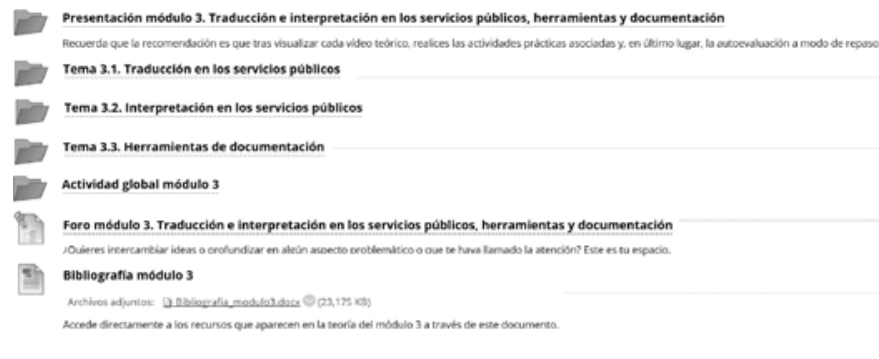

Figura 5: Aspecto general del módulo 3

En lo que respecta a la evaluación, se incluyen posibles respuestas para estos ejercicios, que pueden encontrarse en el lugar correspondiente al tipo de actividad en cuestión: al final del material audiovisual en el que se proponen las actividades, en los apartados del foro identificados mediante el título del ejercicio o, en el caso de las autoevaluaciones, el usuario obtiene la plantilla de respuestas al completar y finalizar el envío.

Cada uno de los módulos tiene asignado un foro de discusión con algunas ideas y temas que incitan el debate e invitan al intercambio de ideas, si bien los alumnos tienen libertad de añadir sus aportaciones propias mediante nuevos temas a medida que profundizan en el curso (Figura 6).

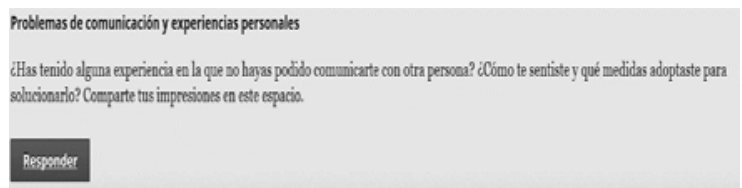

Figura 6: Ejemplo de hilo de debate propuesto por el equipo docente en el foro

Asimismo, se invita a los usuarios a tomar parte en actividades conjuntas a través de sus cuentas de Twitter. Para estas últimas, se potencian al máximo las posibilidades que ofrece esta red social mediante la creación de hilos y hashtags que persiguen involucrar a los usuarios de forma activa y fomentar la visibilidad del curso entre el público general (Figura 7).

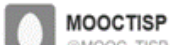

Señala la que consideres la principal dificultad a la que se enfrentan traductores e intérpretes de los servicios públicos. \#TISP.

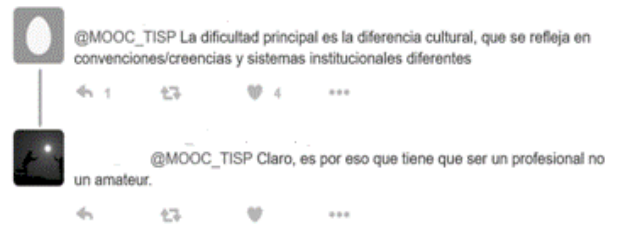

Figura 7: Incorporación de Twitter a las actividades del MOOC y participación de alumnos

Al finalizar el curso se incluye una encuesta de satisfacción que debe completarse para obtener un certificado de realización del MOOC, directamente descargable a través de la plataforma. Esta encuesta se centra en diferentes aspectos:

- Datos generales sobre el perfil del alumnado (edad, país, estudios previos, lenguas de trabajo, situación laboral, conocimientos previos, dedicación diaria, interés en otro curso similar).

- Satisfacción con el contenido del curso (grado de especificidad de los contenidos cubiertos, calidad, aplicabilidad práctica de los conocimientos adquiridos, aspectos estudiados en cada tema, definiciones y explicaciones proporcionadas, bibliografía recomendada).

- Plataforma utilizada: dificultades técnicas, nivel de satisfacción, acceso a los materiales, descarga de contenidos.

- Actividades y autoevaluaciones: nivel de utilidad, aplicabilidad considerando los contenidos teóricos, utilidad del foro de debate.

- Otros aspectos: distribución temporal y tiempo para realizar las actividades, nivel de satisfacción general, dificultades, expectativas, temas o aspectos más interesantes/mejor valoradas, valoración general y sugerencias.

\section{RESULTADOS DE CALIDAD}

En la actualidad contamos con 134 personas inscritas, aunque sólo 20 personas (26 \%) han realizado todas las actividades y lo han evaluado a través de la misma aplicación del curso. Los países de procedencia de los alumnos son variados: Argentina, China, Colombia, España, India, Francia, Serbia y Siria.

Con respecto a sus calificaciones subrayamos las notas altas obtenidas en las actividades de autoevaluación, de 8 a 10, y las medias finales, que oscilan entre 8 y 9,5 (con medias de 8; 8,44; 8,66; 9; 9, 33 y 9,55).

En cuanto al nivel de satisfacción, destacamos su valoración del curso considerando determinados aspectos en el contexto de su propia necesidad formativa en Traducción e Interpretación en los Servicios Públicos:

- Calidad del contenido (pregunta 16 de la encuesta final; Figura 8):

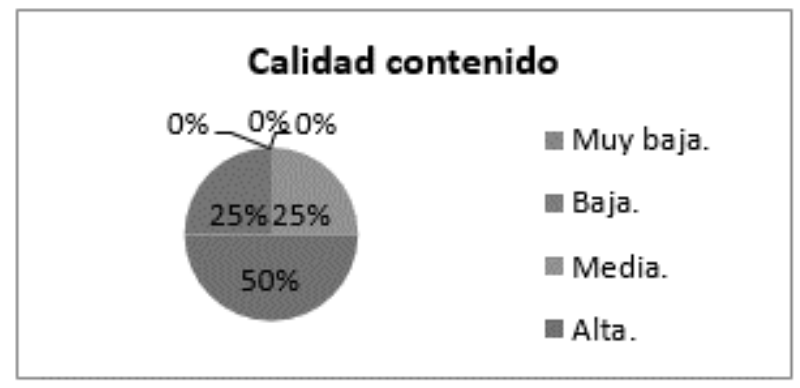

Figura 8: Valoración de la calidad del contenido 
- Aplicabilidad práctica de los conocimientos adquiridos a su propia formación, trabajo, necesidad de información en general (75 \% alta y $25 \%$ moderada) (pregunta 17 de la encuesta final; Figura 9):

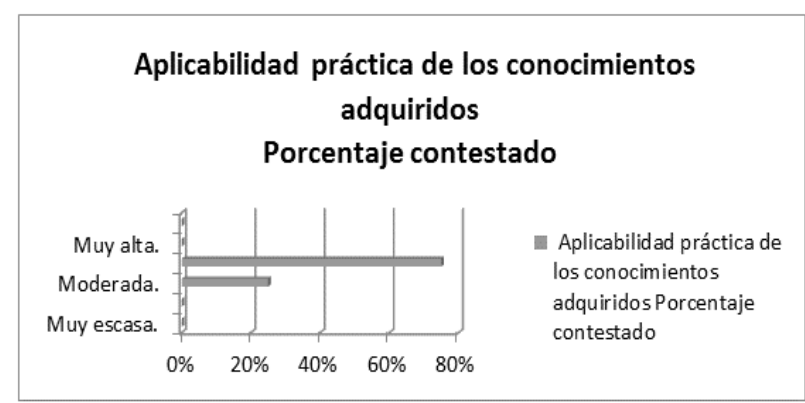

Figura 9: Valoración de la aplicabilidad del contenido

- Nivel de utilidad y adecuación de los aspectos tratados en cada tema considerando la necesidad de formación básica en el campo de la Traducción e Interpretación en los Servicios Públicos (75 \% interesantes y 25 \% muy interesantes; Figura 10):

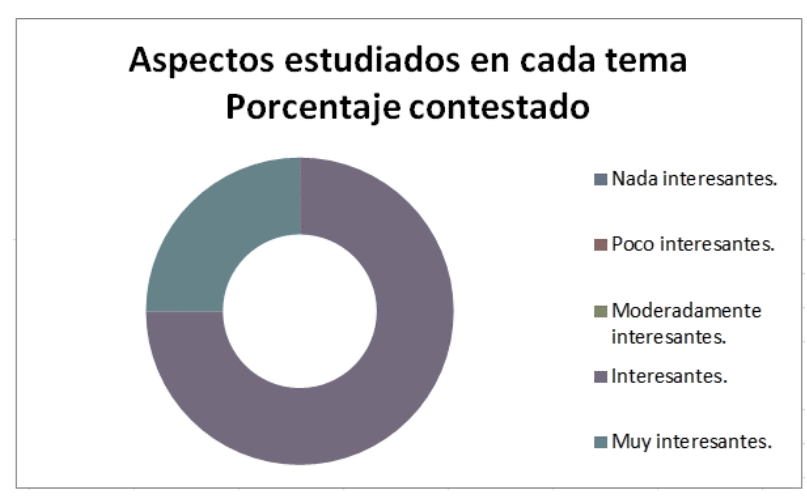

Figura 10: Valoración de la adecuación del contenido de cada tema

Si algunos de los puntos fuertes del curso son el tipo de enseñanza en sí, la selección del contenido y el tipo de actividades (variadas, formato vídeo), algunos puntos débiles señaladas durante la evaluación piloto por alumnado son los siguientes aspectos:

- Duración prevista, aunque también existe la posibilidad de que realice al ritmo del propio usuario.

- Cantidad de vídeos y duración de algunos.

- Atención al estudiante mediante email sin necesidad de realizar entregas de tareas y feedback constante del profesor.

- Baja tasa de finalización. En este caso no podemos hablar de tasa de abandono ya que el curso sigue activo y la participación es activa.

En el caso de los primeros tres aspectos podríamos indicar que se trata de aspectos que una parte del alumnado y el profesorado que lo han pilotado los consideran también como aspectos positivos ya que permiten la flexibilidad del proceso de aprendizaje. Por otro lado, la baja tasa de finalización indicada en el momento de redacción de este artículo, en acorde con otros MOOC en general (Gómez-Serranillos, 2017), se puede explicar por varios motivos como pueden ser:

- $\quad$ Novedad del tema.

- Duración/ plazo amplio de realización.

- $\quad$ Fecha reciente de lanzamiento y el hecho de que el curso sigue abierto en cuanto a inscripción y realización. En realidad, la tasa de realización es creciente.

- Gratuidad, que, según Gómez-Serranillos en un artículo del periódico El Mundo (2017), “se convierte en un arma de doble filo": por un lado, facilita la formación y por otro, no implica compromiso. Según estudios de la Escuela Europea de Dirección de Empresa, aproximadamente el $90 \%$ de los alumnos no finalizan los MOOC.

Como ya indicábamos, actualmente el MOOC está siendo ampliado y adaptado a otras lenguas y culturas (árabe, chino, ruso, francés, inglés).

\section{CONCLUSIONES}

Como conclusiones prácticas podríamos subrayar la utilidad del curso y su impacto, especialmente académico, aunque también social por su aplicabilidad formativa, su carácter gratuito y su potencial para cubrir necesidades formativas específicas en el campo de la Traducción e Interpretación en los Servicios Públicos. En este sentido, utilizando el español como lengua de trabajo, este curso práctico ofrece los siguientes beneficios:

- Promoción de un aprendizaje activo y colaborativo mediante distintos tipos de actividades utilizando diversas herramientas específicas.

- Interacción, comunicación, seguimiento y apoyo a los participantes a través de tutoriales en línea, fórums, correo electrónico, wikis, blogs, etc.

- Contribución para responder a las necesidades de formación de personas que trabajan como traductores/intérpretes/mediadores.

- Contribución a la sociedad del conocimiento ofreciendo formación e información sobre las necesidades lingüísticas en los servicios públicos, la comunicación institucional e intercultural y la importancia de una transmisión eficaz de la información.

Terminamos animando a todos los lectores a seguir el MOOC “¡Iníciate en la Traducción e Interpretación en los servicios públicos!” y a continuar desarrollando otros cursos.

\section{REFERENCIAS}

Álvarez Álvarez, S., Adrada Rafael, C., Bueno García, A., Cuéllar Lázaro, C., López Arroyo, B., Arnáiz Uzquiza, V., Sánchez Nieto, M.T., Fernández Antolín, M.J., Tarancón Álvaro, B. Sánchez Martínez, C., Ramiro Alcántara, J., Cuervo Cuervo, A. y Barrio Muñiz, R. (2016) Primeros pasos por el universo MOOC: planificación y diseño de cursos de traducción y lenguas 
extranjeras. Proyectos de innovación docente - Informe de seguimiento. 1-6.

Recuperado de

http://uvadoc.uva.es/bitstream/10324/16944/1/ANEXO\% 20IVAlvarez\%20Alvarez1516.pdf

Álvaro Aranda, C. y Valero-Garcés, C. (2017) Enseñanza, nuevas tecnologías e innovación en la TISP: introducción al MOOC ¡Atrévete a traducir y a interpretar en los servicios públicos! En C. Valero Garcés et al. (Eds.) Superando límites en Traducción e Interpretación en los Servicios Públicos. Alcalá de Henares, España: Servicio de Publicaciones Universidad de Alcalá.

Cabero Almenara, J., Llorente Cejudo, M.C., y Vázquez Martínez, A.I. (2014) Las tipologías de MOOC: su diseño e implicaciones educativas. Profesorado: Revista de currículum y formación del profesorado, 18 (1), 1326. Recuperado de http://hdl.handle.net/10481/31663

Gómez-Serranillos, M. J (31.mayo 2017) De cómo la formación online y gratuita cambiará el futuro de la Universidad. El Mundo. Recuperado de http://www.elmundo.es/f5/campus/2017/05/31/592db90d e5fdea4f2e8b45e0.html

Marco Cuenca, G., Arquero Avilés, R., Ramos Simó, F., y Cobo Serrano, S. (2010) Análisis de características de los Cursos en Línea Masivos y Abiertos (MOOCs): propuesta de aplicación en escenarios de aprendizaje en el área de Documentación. I Congreso Virtual de Innovación Didáctica al Servicio del Docente y Profesional de Ciencias Documentales. Recuperado de
https://www.researchgate.net/publication/266021624_An alisis_de_caracteristicas_de_los_Cursos_en_Linea_Masi vos_y_Abiertos_MOOCs_propuesta_de_aplicacion_en_e scenarios_de_aprendizaje_en_el_area_de_Documentacio $\mathrm{n}$

Méndez García, C. (2013) Diseño e implementación de cursos abiertos masivos en línea (MOOC): expectativas y consideraciones prácticas. Revista de Educación a Distancia, $39 . \quad$ Recuperado de http://www.um.es/ead/red/39

MOOC TISP. Youtube. (6 julio 2017). ¡Iníciate en la Traducción e Interpretación en los Servicios Públicos! [Video]. Vídeo de presentación del curso MOOC Atrévete a Traducir y a Interpretar en los Servicios Públicos. Recuperado de https://www.youtube.com/watch?v=UsK0tMM2gE\&rel=0

OpenEducation. Plataforma para cursos en formato abierto. Recuperado de https://openeducation.blackboard.com

Red de Másteres Europeos de Traducción EMT. Competence Wheel (2009) EMT. Master's in Translation. Translation Competences. Recuperado de https://ec.europa.eu/info/european-masters-translationemt_en

Roig Vila, R., Mengual Andrés, S., y Suárez Guerrero, C. (2014) Evaluación de la calidad pedagógica de los MOOC. Revista de Currículum y Formación de Profesorado, 18 (1). Recuperado de: http://www.redalyc.org/articulo.oa?id=56730662003 